\title{
Preliminary comparison of 3D synthetic aperture imaging with Explososcan
}

Rasmussen, Morten Fischer; Hansen, Jens Munk; Ferin, Guillaume; Dufait, Remi; Jensen, Jørgen Arendt

Published in:

Proceedings of SPIE

Link to article, DOI:

$10.1117 / 12.913925$

Publication date:

2012

Link back to DTU Orbit

Citation (APA):

Rasmussen, M. F., Hansen, J. M., Ferin, G., Dufait, R., \& Jensen, J. A. (2012). Preliminary comparison of 3D synthetic aperture imaging with Explososcan. In Proceedings of SPIE (Vol. 8320, pp. 83200F-9). SPIE International Society for Optical Engineering. https://doi.org/10.1117/12.913925

\section{General rights}

Copyright and moral rights for the publications made accessible in the public portal are retained by the authors and/or other copyright owners and it is a condition of accessing publications that users recognise and abide by the legal requirements associated with these rights.

- Users may download and print one copy of any publication from the public portal for the purpose of private study or research.

- You may not further distribute the material or use it for any profit-making activity or commercial gain

- You may freely distribute the URL identifying the publication in the public portal 


\title{
Preliminary comparison of 3D synthetic aperture imaging with Explososcan
}

\author{
Morten Fischer Rasmussen ${ }^{a}$, Jens Munk Hansen ${ }^{a}$, Guillaume Férin ${ }^{b}$, Rémi Dufait $^{b}$ and \\ Jørgen Arendt Jensen ${ }^{a}$ \\ ${ }^{a}$ Center for Fast Ultrasound Imaging, Dept. of Elec. Eng. Bldg. 349, \\ Technical University of Denmark, DK-2800 Kgs. Lyngby, Denmark. \\ ${ }^{b}$ Vermon S.A., Avenue du Général Renault, 37000 Tours, France.
}

\begin{abstract}
Explososcan is the 'gold standard' for real-time 3D medical ultrasound imaging. In this paper, 3D synthetic aperture imaging is compared to Explososcan by simulation of 3D point spread functions. The simulations mimic a 32x32 element prototype transducer. The transducer mimicked is a dense matrix phased array with a pitch of $300 \mu \mathrm{m}$, made by Vermon. For both imaging techniques, 289 emissions are used to image a volume spanning $60^{\circ}$ in both the azimuth and elevation direction and $150 \mathrm{~mm}$ in depth. This results for both techniques in a frame rate of $18 \mathrm{~Hz}$. The implemented synthetic aperture technique reduces the number of transmit channels from 1024 to 256, compared to Explososcan. In terms of FWHM performance, was Explososcan and synthetic aperture found to perform similar. At $90 \mathrm{~mm}$ depth is Explososcan's FWHM performance $7 \%$ better than that of synthetic aperture. Synthetic aperture improved the cystic resolution, which expresses the ability to detect anechoic cysts in a uniform scattering media, at all depths except at Explososcan's focus point. Synthetic aperture reduced the cyst radius, $R_{20 \mathrm{~dB}}$, at $90 \mathrm{~mm}$ depth by $48 \%$. Synthetic aperture imaging was shown to reduce the number of transmit channels by four and still, generally, improve the imaging quality.
\end{abstract}

Keywords: medical ultrasound, three-dimensional imaging, synthetic aperture, explososcan

\section{INTRODUCTION}

In conventional ultrasound, images are produced by sequentially acquiring and beamforming scan lines, followed by a scan line conversion. For at setup using $N$ scan lines per image the achievable frame rate, $f_{r}$, when acquiring to a depth of $D$, is then

$$
f_{r}=\frac{c}{2 D N}
$$

In 3D ultrasound imaging, an entire volume is imaged. The extension from 2D to 3D greatly increases the number of lines to be acquired, e.g. from 100 to $100^{2}$ lines. This increase leads to a proportional decrease in frame rate. Using conventional imaging, the achievable frame rate is too low to capture the dynamics of the anatomy, e.g. the heart. This was addressed by von Ramm and Smith ${ }^{1-3}$ by applying a parallel beamforming technique, dubbed Explososcan, to 3D imaging.

In Explososcan, multiple lines are beamformed per transmission, thus increasing the achievable frame rate. If, for instance, $6 \times 6$ lines are beamformed per transmission instead of just one, the frame rate increases by a factor of 36. Besides increasing the frame rate, Explososcan introduces some undesirable effects. In order to cover the extra scan lines, must the emission beam insonify a larger volume, and a less focused beam is therefore needed. This leads to a larger point spread function main lobe. The further away from an emission beam a line is beamformed, the less energy the beamformed area receives from the emission beam, and the darker it appears in the image. This is an amplitude modulation of the image lines, with peak intensity at lines co-aligned with emission beams, and lower intensity further away from the emission beams. A second effect can be observed. Scan lines beamformed from a the same emission exhibit higher correlation with one another, than do lines not beamformed from a the same emission. Together these two effects are termed the "boxing effect".

An alternative technique called synthetic aperture, ${ }^{4-8}$ exists. The synthetic aperture technique synthesizes a larger aperture from measurements carried out using apertures located at different positions. Synthetic aperture 
can be divided into two major groups; the synthetic receive aperture, where a larger receive aperture is synthesized, and the synthetic transmit aperture, ${ }^{9}$ where a larger transmit aperture is synthesized. The basic principle of synthetic transmit aperture is that a single point source emits a spherical wave, insonifying the entire image region. This is repeated for all available sources, each located at different locations. After each transmission, all elements in the receive aperture are sampled. Each sampled data set is used for beamforming a low resolution image, which is dynamically receive focused. Summing all low resolution images results in a fully focused high resolution image. The high resolution image is, thus, dynamically focused in both receive and transmit. The end effect is a synthesized transmit aperture, with the point sources as elements and a dynamic transmit focus. In this paper is synthetic transmit implemented and synthetic aperture will refer to synthetic transmit aperture.

The imaging quality of synthetic aperture is compared to Explososcan, when doing 3D imaging with a 2D phased array transducer. The comparison of the two imaging techniques is based on performance metrics applied to their respective point spread function.

The organization of this paper is as follows: First, the applied performance metrics are introduced. Next, it is described how Explososcan and synthetic aperture are implemented and the simulation setup is described. In the results section, a visual comparison of two point spread functions is made and the acquired performance metrics as a function of depth are presented. Finally, some concluding remarks are given.

\section{PERFORMANCE METRICS}

Metrics quantifying different quality features can be used to compare the two imaging techniques. In this paper, both a detail resolution and a cystic resolution metric are applied to the point spread functions. Both are introduced in detail in the following.

\subsection{Detail resolution}

The detail resolution is how close two identical point scatters in a non-reflective medium can be located to each other and still be distinguished. An often used measure for when two scatterer can be resolved is the size of the point spread function main lobe, i.e. the full width at half maximum (FWHM).

The FWHM is determined in both the azimuth and elevation direction of the point spread function C-scan. A single number is achieved by averaging the two measures.

\subsection{Cystic resolution}

The cystic resolution describes the ability to detect an anechoic cyst in a uniform scattering medium. Specifically, the cystic resolution gives the intensity at the center of an anechoic cyst of a given size relative to its surrounding uniformly back-scattering medium. ${ }^{10}$ The lower the intensity at the center of the cyst, i.e. the darker it appears in the image for a given cyst size, the better imaging performance of the system. This metric mimics an often seen situation in medical ultrasound where the object of interest is a weakly reflecting tissue, such as a blood vessel or a cyst, in a back-scattering medium. For this reason, is cystic resolution assumed to be a more important performance metric than the FWHM. The relative intensity (RI) of the anechoic cyst was shown by Ranganathan and Walker, ${ }^{11}$ to be quantized as the clutter energy to total energy ratio,

$$
\operatorname{RI}(R)=\sqrt{\frac{E_{\text {out }}(R)}{E_{\text {tot }}}}=\sqrt{1-\frac{E_{\text {in }}(R)}{E_{\text {tot }}}},
$$

where $E_{\text {in }}$ is the signal energy inside a circular region with radius, $R$, centered on the peak of the point spread function, $E_{t o t}$ is the total point spread function energy, and $E_{\text {out }}$ is the point spread function energy outside the circular region. To achieve a single number from the $\mathrm{RI}(R)$-curve, one either determines the relative intensity for a single cyst radius, or determines the required radius to achieve a certain relative intensity, e.g. the cyst radius at which the intensity at center of the cyst is $20 \mathrm{~dB}$ lower than its surroundings, written as $R_{20 \mathrm{~dB}}$.

The cystic resolution is in this work measured in the point spread function $\mathrm{C}$-scan and is presented both as a full $\mathrm{RI}(R)$-curve and as a single cyst radius giving a relative intensity of $20 \mathrm{~dB}, R_{20 \mathrm{~dB}}$. 


\section{METHODS}

\subsection{Explososcan}

As described in the introduction, the procedure of beamforming several lines per emission introduces an undesirable boxing effect consisting of two effects; firstly, an intensity modulation of the beamformed image lines, and secondly, scan lines beamformed from the same emission exhibit a higher correlation with one another, than do lines not beamformed from the same emission. If the line intensity modulation factor is known it can be compensated for. The intensity modulation factor could be estimated from simulations, but this is not implemented. The correlation effect can to some extent be compensated for by overlapping lines beamformed from neighboring emissions. When overlapping scan lines, each line has to be weighted in order to ensure a uniform intensity in the image. It is intuitive to have the largest scan line weight when the scan line and its emission beam are parallel, and a lower weight as the angle between them increases. In this work, it is chosen to have the weight of each scan line to be inversely proportional with the angle difference between the scan line and its emission beam. If we define the distance measure

$$
\mathrm{d}(\ell, e)=\sqrt{\left(\theta_{\ell}-\theta_{e}\right)^{2}+\left(\phi_{\ell}-\phi_{e}\right)^{2}},
$$

where $\ell$ is a scan line in a given direction, $e$ is the emission beam from which $\ell$ was beamformed, $\phi$ is the azimuth angle and $\theta$ the elevation angle of the line, the weight function then becomes

$$
w(\ell, e)=\left\{\begin{array}{ll}
1 & \text { if } \quad \mathrm{d}(\ell, e)=0 \\
\frac{1 / \mathrm{d}(\ell, e)}{Z(\ell)} & \text { else }
\end{array},\right.
$$

where $Z(\ell)$ is a normalization factor, which makes sure the weights of all lines overlapping with line $\ell$ sums to one.

In Fig. 1, the effect of the implemented correlation compensation is seen on a $\mathrm{C}$-scan measurement of a tissue mimicking phantom. In all four sub-figures contains the $\mathrm{C}$-scans the same number of resulting lines, but the number of lines overlapping differs. In Fig. 1(a) the C-scan is seen without correlation compensation and in Fig. 1(b)-(d) the correlation compensation is increased one step at a time. Each step of correlation compensation is dubbed 1st level, 2nd level and 3rd level of correlation compensation, respectively. The number of lines beamformed per emission is increased from $6 \times 6$ in Fig. 1(a), through $8 \times 8,10 \times 10$ and $12 \times 12$, in Fig. 1(b)-(d). As the correlation compensation increases, the $\mathrm{C}-$-scan gets more blurred and the dynamic range decreases. For the Explososcan implementation, the 1st level of correlation compensation is chosen. The 1st level of correlation compensation is a compromise between a very evident boxing effect, when no correlation compensation is applied, and a blurry image when the 3rd level of correlation compensation is applied.

Adding extra scan lines goes against the original Explososcan idea of decreasing the number of beamformers. But the new scan lines could be beamformed by using the parallel beamforming approximation, described by von Ramm and colleagues in, ${ }^{3}$ and thereby keeping the computational cost low.

\subsection{Synthetic aperture}

In the standard version of synthetic aperture, each source emits an unfocused spherical wave. By using multiple transducer elements in transmit, a virtual source can be imitated at the focus point. This leads to an increase in emitted energy and penetration depth. If the virtual source is located directly in front of the active aperture, the synthesized transmit aperture is smaller than the physical transducer. This is illustrated in Fig. 2(a). The larger the sub-aperture, the smaller the synthesized aperture. For 2D imaging, the maximum number of synthesized transmit sources can be found by the following relation,

$$
N_{\text {vs }}=N_{\text {phys }}-N_{\text {act }}+1
$$

where $N_{\mathrm{vs}}$ is the number of virtual sources in the synthesized transmit aperture, $N_{\text {phys }}$ is the number of physical elements, and $N_{\text {act }}$ is the number of active physical elements used for the emissions. If 16 elements are used for each dimension of the sub-aperture and the physical transducer has a size of $32 \times 32$ elements, the synthesized 


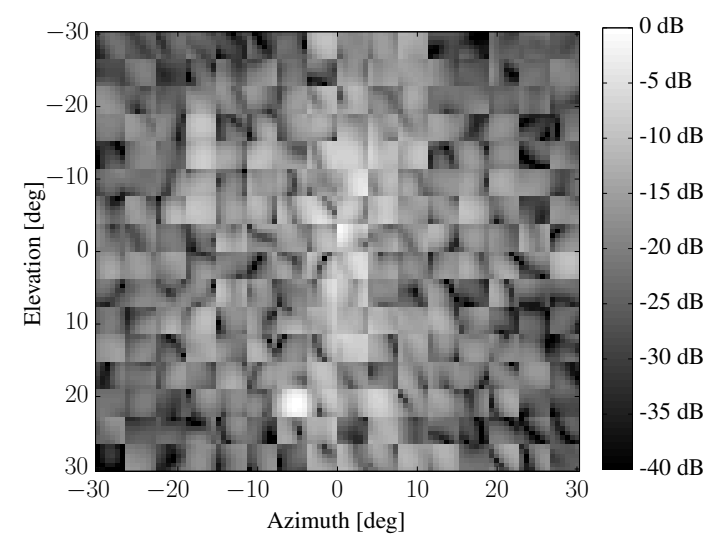

(a) No correlation compensation; $6 \times 6$ lines beamformed per emission.

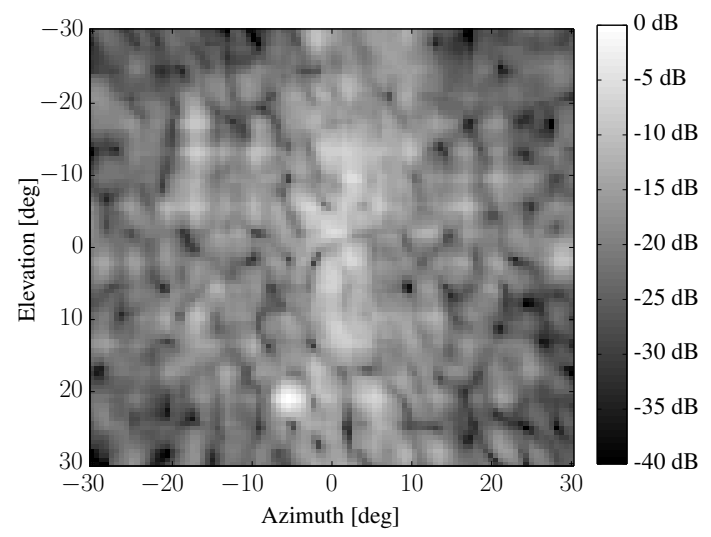

(c) 2nd level of correlation compensation; $10 \times 10$ lines beamformed per emission.

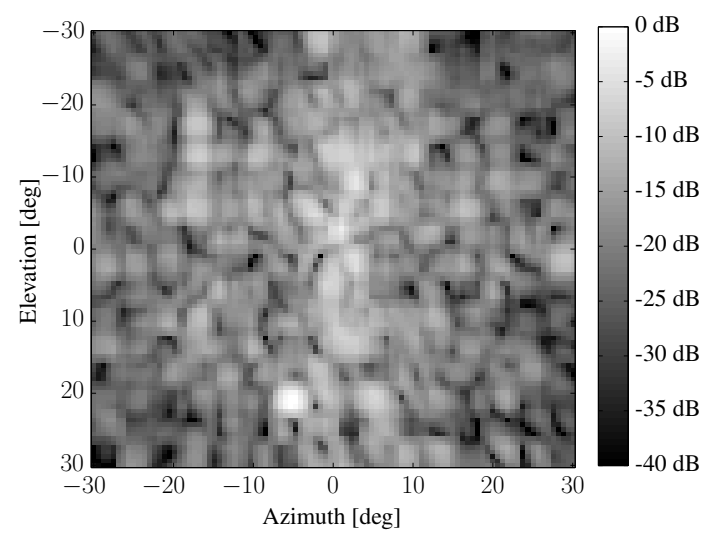

(b) 1st level of correlation compensation; $8 \times 8$ lines beamformed per emission.

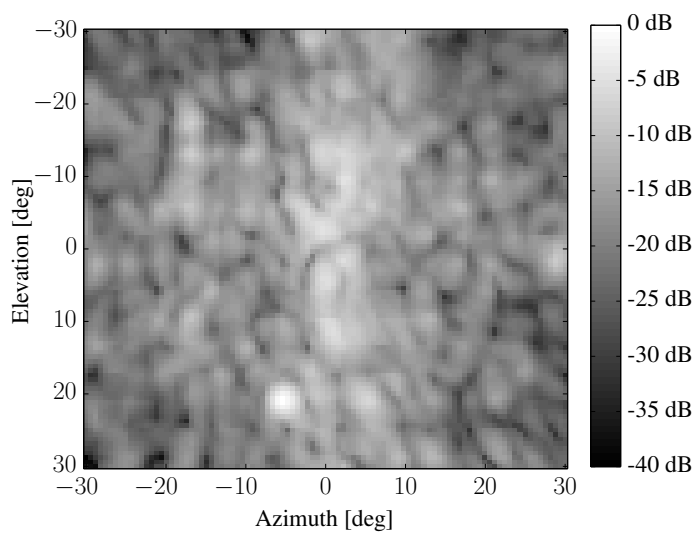

(d) 3rd level of correlation compensation; $12 \times 12$ lines beamformed per emission.

Figure 1: Explososcan correlation compensation for a C-scan measurement of a tissue mimicking phantom. The transducer parameters can be seen in Table 1 .

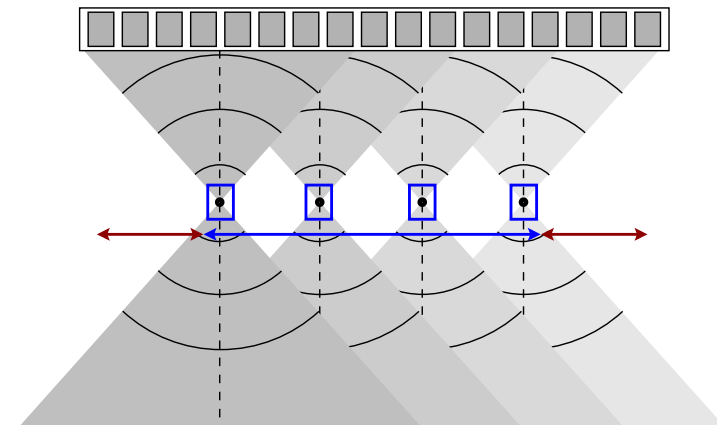

(a) Traditional synthetic aperture. The virtual source is centered on each sub-aperture.

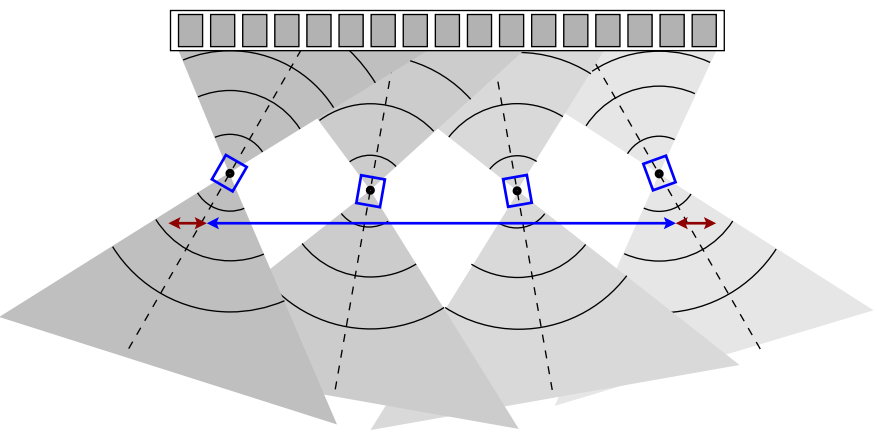

(b) Synthetic aperture with beam steering. The virtual source is tilted to the sides when the active aperture is translated.

Figure 2: Synthetic aperture virtual source setup. The gray array in the top is the physical aperture, the thick dots the virtual sources, and the array of boxes around the virtual sources the synthesized aperture. Four emissions are shown, each with eight active elements. The center arrow indicates the synthesized aperture size. 
transmit aperture contains $17 \times 17$ elements. This is little more than half the size of the physical transducer, and the transmit $\mathrm{f}$-number is therefore approximately doubled, compared to a full $32 \mathrm{x} 32$ element transmission.

A method to partially compensate for the smaller synthesized transmit aperture is to spread the virtual sources apart by tilting the emission beam proportionally with the active aperture location on the transducer. This way, the synthesized aperture is increased in size. This illustrated in Fig. 2(b).

Synthetic aperture imaging is in this work implemented with emission beam steering.

\section{SIMULATION SETUP}

All simulations are carried out using Field $\mathrm{II}^{12,13}$ and beamformed using BFT3. ${ }^{14}$ The transducer used in the simulations mimics a prototype transducer soon to be used for $3 \mathrm{D}$ synthetic aperture measurements. It is a $2 \mathrm{D}$ phased array transducer, made by Vermon, (Vermon S.A., Tours, France), with a layout of 35x32 elements, of which row 9,18 and 27 are inactive, effectively giving $32 \times 32$ elements. The transducer parameters can be seen in Table 1. The point spread function is simulated by imaging a volume containing a single point scatter. The point

Table 1: Transducer parameters

\begin{tabular}{lcc}
\hline \hline Parameter name & Notation & Value \\
\hline Center frequency & $f_{c}$ & $3.5 \mathrm{MHz}$ \\
Transducer pitch $-\mathrm{x}$ & $d_{x}$ & $300 \mu \mathrm{m}$ \\
Transducer pitch $-\mathrm{y}$ & $d_{y}$ & $300 \mu \mathrm{m}$ \\
Total number of elements $-\mathrm{x}$ & $N_{t o t \_x}$ & 32 \\
Total number of elements $-\mathrm{y}$ & $N_{\text {tot_y }}$ & 35 \\
Inactive rows & - & $9,18,27$ \\
Number of active elements $-\mathrm{x}$ & $N_{x}$ & 32 \\
Number of active elements $-\mathrm{y}$ & $N_{y}$ & 32 \\
\hline \hline
\end{tabular}

scatterers are placed in front of the transducer at depths ranging from $40 \mathrm{~mm}$ to $150 \mathrm{~mm}$. The emission beam is controlled similarly to what is used for phased array imaging, and the coordinate system used is the one defined by Smith et al. ${ }^{2}$ For both Explososcan and synthetic aperture, the transmit aperture is apodized with a Tukey window, and for receive apodization, a Hanning window is applied.

\subsection{Explososcan}

The implemented Explososcan technique uses, in both transmit and receive, all 1024 available elements of the 32x32 element transducer. Explososcan is implemented with 17 emissions in both the azimuth and elevation direction, which results in 289 emissions per imaged volume. As discussed in Sec. 3.1, to counteract the boxing effect of Explososcan, some of the scan lines are overlapped. $8 \times 8$ lines are beamformed per emission, but $6 \times 6$ lines appear in the volume, resulting in $102 \times 102$ total lines in the volume. The beamformed lines span from $-30^{\circ}$ to $30^{\circ}$ in both directions, and the emission beams are spread evenly within this span. The focus point is placed at $40 \mathrm{~mm}$ range, which gives a transmit f-number ranging from 4.2 to 4.8 , depending on the emission beam angle.

\subsection{Synthetic aperture}

For synthetic aperture, there is for each emission used an active aperture of $16 \times 16$ elements in transmit, and all 1024 elements in receive. This results in 256 active elements in transmit, which is one fourth of what is used for the Explososcan implementation. The active aperture is translated between each emission, one element in either the $\mathrm{x}$ - or $\mathrm{y}$-direction. The active aperture is therefore translated 17 times in both the $\mathrm{x}$ - and the $\mathrm{y}$-direction, giving a total of 289 emissions. This is the same number of emissions per volume as for the Explososcan implementation. The emission beam is tilted from $-15^{\circ}$ to $15^{\circ}$, proportional to the active aperture position on the transducer. The virtual source is placed at $10 \mathrm{~mm}$ range, which gives a transmit $\mathrm{f}$-number ranging from 2.0 to 2.2 . For each emission $102 \times 102$ scan lines are beamformed, spanning from $-30^{\circ}$ to $30^{\circ}$ in both directions. This is the same as the number of resulting lines in the Explososcan implementation. 
When imaging to a depth of $150 \mathrm{~mm}$, the 289 emission per volume results for both synthetic aperture and Explososcan in a frame rate of approximately $18 \mathrm{~Hz}$.

\section{RESULTS}

In Fig. 5, a point spread function example of Explososcan and synthetic aperture is seen, and can be visually inspected and compared. The point spread function is located at $90 \mathrm{~mm}$ depth and $0^{\circ}$ azimuth and elevation tilt angle. In the azimuth plane are the side-lobes larger than in the elevation plane. This is due to three inactive rows of elements on the transducer, all parallel to the elevation plane. Synthetic and Explososcan appears to have approximately the same main lobe size, but synthetic aperture clearly has smaller side-lobes. This is especially evident in the elevation plane.

In Fig. 3 is the FWHM as a function of depth seen. Even though Explososcan uses four times more transmit channels than synthetic aperture, is the FWHM performance of the two techniques similar. Explososcan is seen to perform a little better than synthetic aperture at all depths. The largest performance difference occurs at Explososcan's focus point and the smallest at the deepest depth, $150 \mathrm{~mm}$. At $90 \mathrm{~mm}$ is the FWHM of synthetic aperture $4.7 \mathrm{~mm}$ and $4.36 \mathrm{~mm}$ for Explososcan. In percentage, this is a difference of $7 \%$.

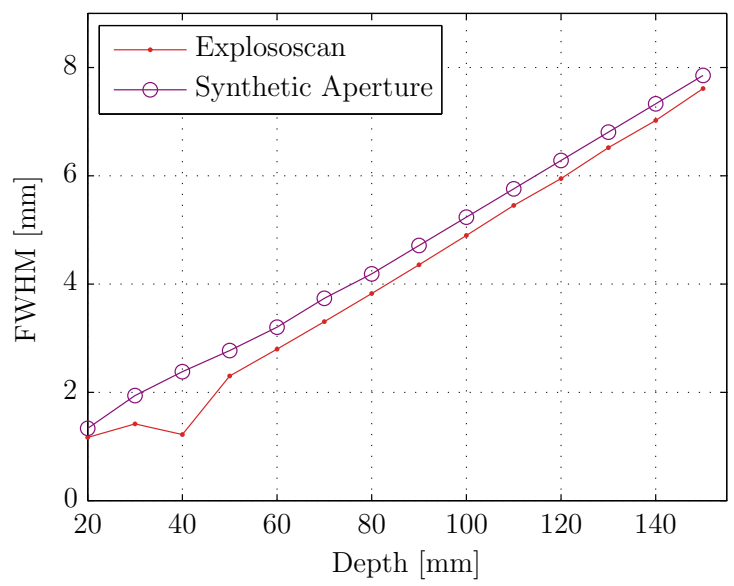

Figure 3: FWHM as a function of depth.

In Fig. 4 is the cystic resolution at $120 \mathrm{~mm}$ depth and the $R_{20 \mathrm{~dB}}$ as a function of depth shown. In Fig. 4(a) is synthetic aperture seen to give a better relative intensity at the cyst center, for all cyst sizes. This means, all anechoic cysts appears darker when imaged with synthetic aperture imaging than with Explososcan. As an example, a $10 \mathrm{~mm}$ anechoic cyst has a relative intensity of $-31 \mathrm{~dB}$ when imaged with synthetic aperture and $-23 \mathrm{~dB}$ when imaged with Explososcan.

The $R_{20 \mathrm{~dB}}$ in Fig. 4(b), increases for both techniques approximately linearly with depth. Explososcan deteriorates more than twice as fast width depth as synthetic aperture, but performs at its focus point a little better than synthetic aperture. Everywhere else outperforms synthetic aperture Explososcan. At $90 \mathrm{~mm}$ depth is the $R_{20 \mathrm{~dB}}$ of synthetic aperture $3.7 \mathrm{~mm}$, and $7.07 \mathrm{~mm}$ for Explososcan. In percentage, decreases synthetic aperture the $20 \mathrm{~dB}$ cyst radius at $90 \mathrm{~mm}$ depth by $48 \%$ compared to Explososcan.

\section{DISCUSSION AND CONCLUSION}

3D synthetic aperture imaging was compared with Explososcan by simulating 3D point spread functions. The implemented Explososcan is partly compensated for its boxing effect. The implemented synthetic aperture technique uses one fourth the number of transmit channels of Explososcan. Both techniques scans a volume to $150 \mathrm{~mm}$ depth with a frame rate of $18 \mathrm{~Hz}$. 


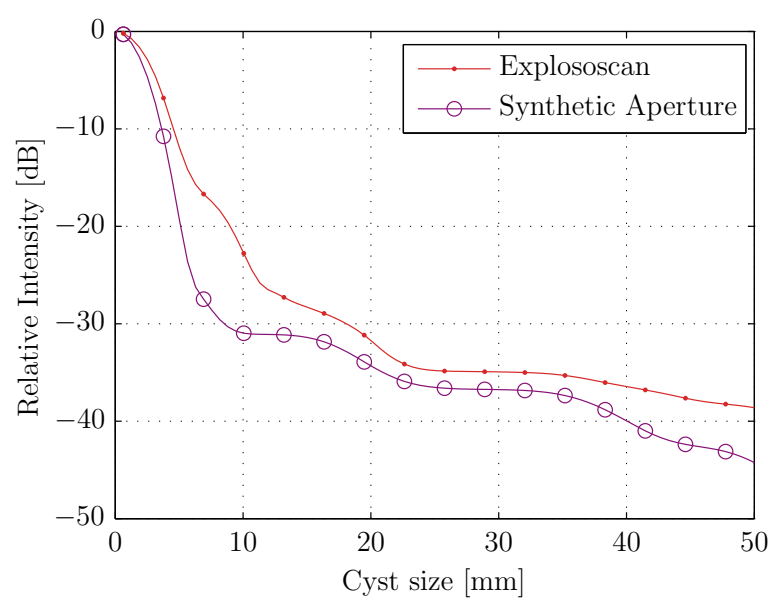

(a) Cystic resolution at $120 \mathrm{~mm}$ depth.

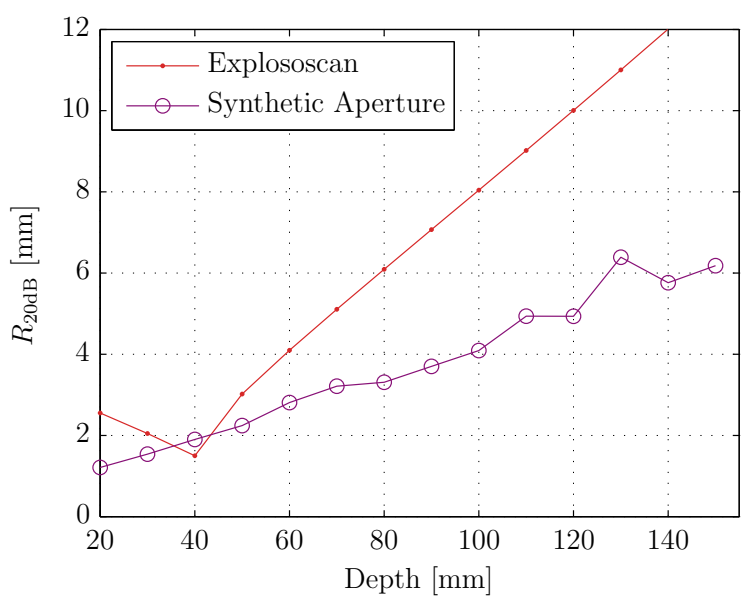

(b) $R_{20 \mathrm{~dB}}$ as a function of depth.

Figure 4: The cystic resolution.

In terms of FWHM, performs Explososcan and synthetic aperture almost the same. At $90 \mathrm{~mm}$ depth is Explososcan's FWHM performance $7 \%$ better than that of synthetic aperture. At Explososcan's focus point performed Explososcan better than synthetic aperture, measured in both FWHM and cystic resolution.

Synthetic aperture was found to generally have a better cystic performance than Explososcan. At $120 \mathrm{~mm}$ depth achieves synthetic aperture a better cystic resolution, for all cyst sizes, than Explososcan. The $R_{20 \mathrm{~dB}}$ performance of synthetic aperture was clearly better than Explososcan at all depths except at Explososcan's focus point. Synthetic aperture decreased $R_{20 \mathrm{~dB}}$ at $90 \mathrm{~mm}$ depth by $48 \%$, compared to Explososcan.

There are still many variables to be optimized for the implemented synthetic aperture, including the f-number, the active sub-aperture size, the number of emissions, and amount of emission beam tilt.

From the simulated point spread functions, it is estimated that synthetic aperture achieves a better imaging quality for medical imaging, than Explososcan. This is based mainly on synthetic aperture's better cystic resolution performance.

\section{ACKNOWLEDGMENTS}

This work was financially supported by grant 024-2008-3 from the Danish Advanced Technology Foundation and from BK Medical (BK Medical ApS, Herlev, Denmark).

\section{REFERENCES}

[1] Shattuck, D. P., Weinshenker, M. D., Smith, S. W., and von Ramm, O. T., "Explososcan: A parallel processing technique for high speed ultrasound imaging with linear phased arrays," Journal of the Acoustical Society of America 75, 1273-1282 (1984).

[2] Smith, S. W., Pavy, H. G., and von Ramm, O. T., "High-speed ultrasound volumetric imaging system Part I: Transducer design and beam steering," IEEE Transactions on Ultrasonics, Ferroelectrics, Frequency Control 38, 100-108 (1991).

[3] von Ramm, O. T., Smith, S. W., and Pavy, H. G., "High-speed ultrasound volumetric imaging system Part II: Parallel processing and image display," IEEE Transactions on Ultrasonics, Ferroelectrics, Frequency Control 38, 109-115 (1991).

[4] Flaherty, J. J., Erikson, K. R., and Lund, V. M., "Synthetic aperture ultrasound imaging systems," (United States Patent, US 3,548,642, 1967, Published 22 Dec 1970). 
[5] Burckhardt, C. B., Grandchamp, P.-A., and Hoffmann, H., "An experimental 2 MHz synthetic aperture sonar system intended for medical use," IEEE Transactions on Sonics and Ultrasonics 21, 1-6 (January 1974).

[6] Nagai, K., "A new synthetic-aperture focusing method for ultrasonic $B$-scan imaging by the fourier transform," IEEE Transactions on Sonics and Ultrasonics SU-32(4), 531-536 (1985).

[7] Soumekh, M., [Synthetic aperture radar. Signal processing with MATLAB algorithms], John Wiley \& Sons, Inc., New York (1999).

[8] Jensen, J. A., Nikolov, S., Gammelmark, K. L., and Pedersen, M. H., "Synthetic aperture ultrasound imaging," Ultrasonics 44, e5-e15 (2006).

[9] Chiao, R. Y., Thomas, L. J., and Silverstein, S. D., "Sparse array imaging with spatially-encoded transmits," in [IEEE Ultrasonics Symposium], 1679-1682 (1997).

[10] Vilkomerson, D., Greenleaf, J., and Dutt, V., "Towards a resolution metric for medical ultrasonic imaging," in [Ultrasonics Symposium, 1995. Proceedings., 1995 IEEE], 2, 1405 -1410 vol.2 (nov 1995).

[11] Ranganathan, K. and Walker, W., "Cystic resolution: A performance metric for ultrasound imaging systems," Ultrasonics, Ferroelectrics and Frequency Control, IEEE Transactions on 54, 782 -792 (april 2007).

[12] Jensen, J. A. and Svendsen, N. B., "Calculation of Pressure Fields from Arbitrarily Shaped, Apodized, and Excited Ultrasound Transducers," IEEE Transactions on Ultrasonics, Ferroelectrics, Control 39, 262-267 (1992).

[13] Jensen, J. A., "Field: A Program for Simulating Ultrasound Systems," Medical and Biological Engineering and Computing 4, Supplement 1, Part 1(1), 351-353 (1996).

[14] Hansen, J. M., Hemmsen, M. C., and Jensen, J. A., "An object-oriented multi-threaded software beamformation toolbox," in [SPIE 7968: Vascular Imaging and Ultrasound Beam Forming], 79680Y, SPIE (2011). 


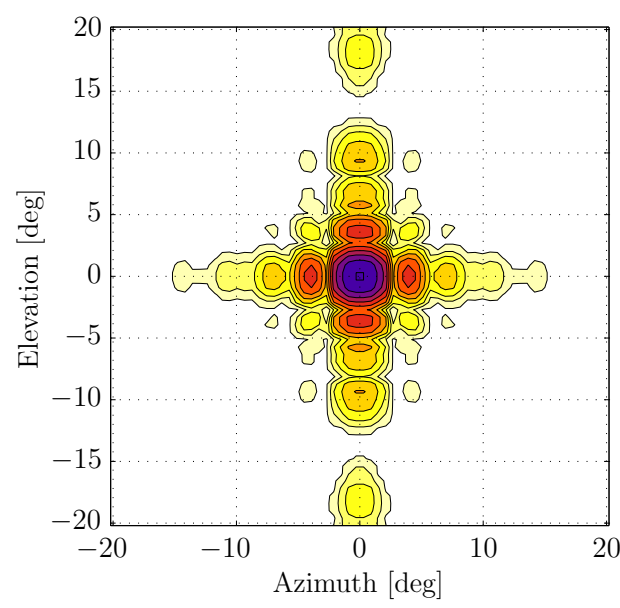

(a) Explososcan C-scan.

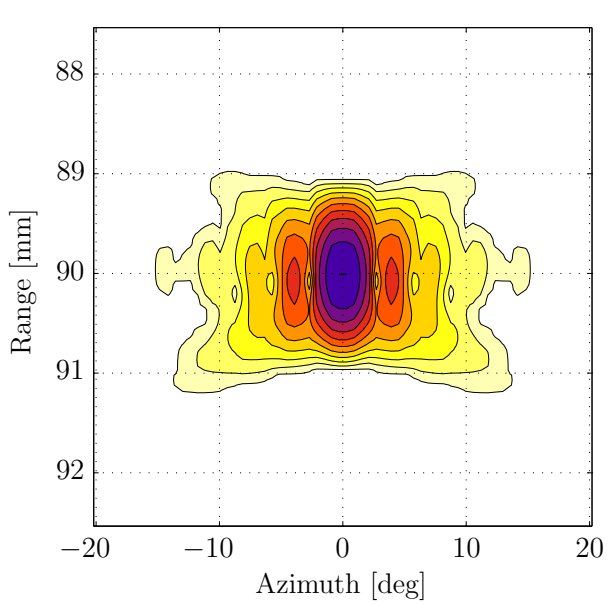

(c) Explososcan azimuth plane.

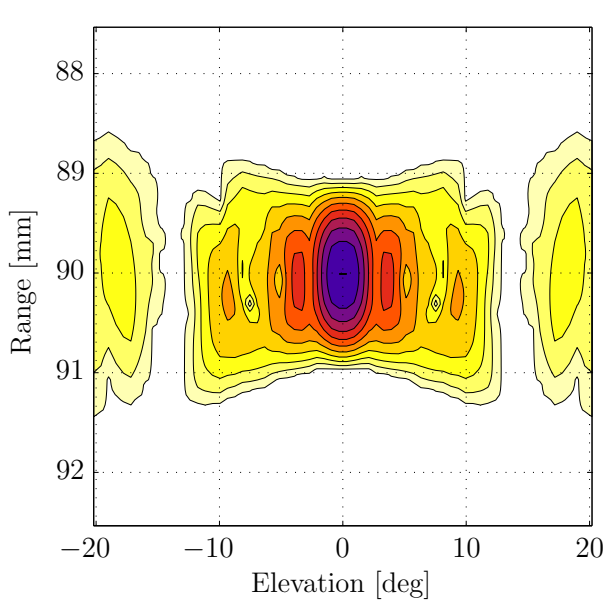

(e) Explososcan elevation plane.

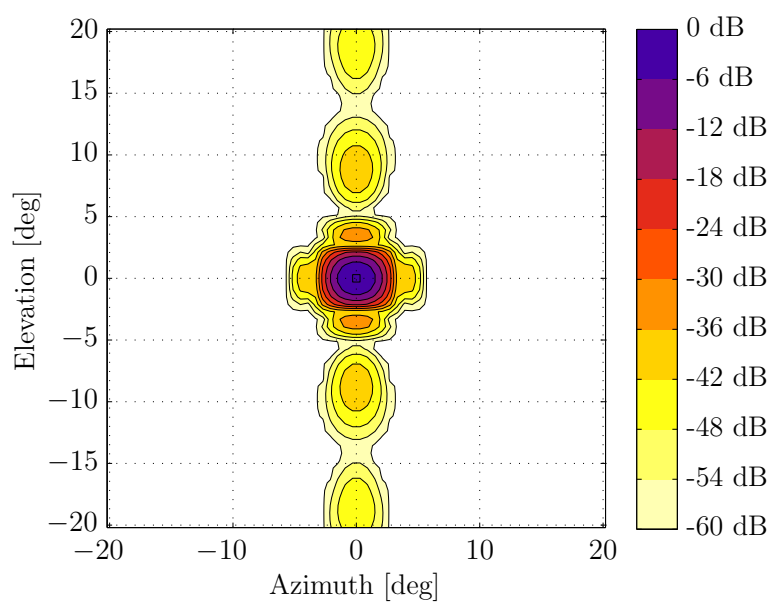

(b) Synthetic aperture C-scan.

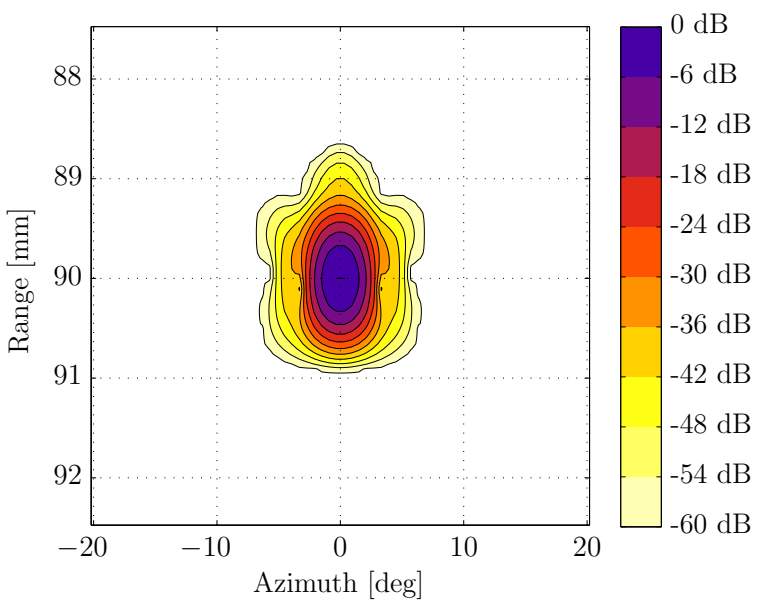

(d) Synthetic aperture azimuth plane.

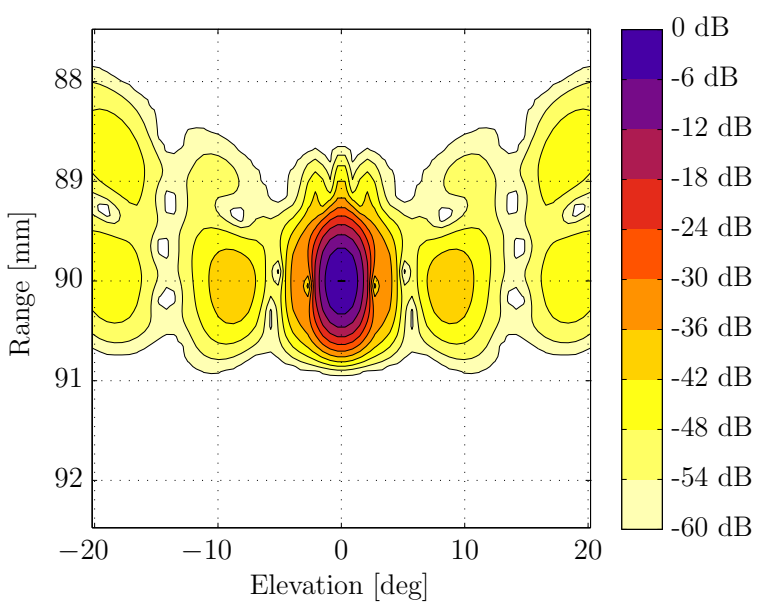

(f) Synthetic aperture elevation plane.

Figure 5: Explososcan and synthetic aperture 3D point spread function sliced into three 2D planes. The point spread functions are observed at $90 \mathrm{~mm}$ depth and $0^{\circ}$ azimuth and elevation tilt angle. The left column is Explososcan and the right column is synthetic aperture. 